Article

\title{
Toward Nonlocal Electrodynamics of Accelerated Systems
}

\author{
Bahram Mashhoon 1,2 \\ 1 Department of Physics and Astronomy, University of Missouri, Columbia, MO 65211, USA; \\ mashhoonb@missouri.edu \\ 2 School of Astronomy, Institute for Research in Fundamental Sciences (IPM), Tehran 19395-5531, Iran
}

Received: 3 November 2020; Accepted: 29 November 2020; Published: 3 December 2020

check for updates

\begin{abstract}
We revisit acceleration-induced nonlocal electrodynamics and the phenomenon of photon spin-rotation coupling. The kernel of the theory for the electromagnetic field tensor involves parity violation under the assumption of linearity of the field kernel in the acceleration tensor. However, we show that parity conservation can be maintained by extending the field kernel to include quadratic terms in the acceleration tensor. The field kernel must vanish in the absence of acceleration; otherwise, a general dependence of the kernel on the acceleration tensor cannot be theoretically excluded. The physical implications of the quadratic kernel are briefly discussed.
\end{abstract}

Keywords: relativity; accelerated systems; nonlocal electrodynamics

\section{Introduction}

Consider an inertial observer $\mathcal{O}$ moving with constant velocity $\mathbf{v}$ in an inertial frame of reference in Minkowski spacetime. The observer's 4-velocity $u^{\mu}=d x^{\mu} / d \tau$ can be expressed in Cartesian coordinates $x^{\mu}=(t, x, y, z)$ as $u^{\mu}=\gamma(1, \mathbf{v})$, where $\tau$ is the observer's proper time, $d \tau=d t / \gamma$, and $\gamma$ is the Lorentz factor. The observer measures the frequency $\omega$ of an incident plane monochromatic electromagnetic wave with propagation vector $k_{0}^{\mu}=\left(\omega_{0}, \mathbf{k}_{0}\right)$, where $\omega_{0}$ is the frequency and $\mathbf{k}_{0}$ is the wave vector with $\omega_{0}=\left|\mathbf{k}_{0}\right|$, as measured by inertial observers $\mathcal{O}_{0}$ at rest in the background inertial frame. As is well known, $\omega$ is given by the relativistic Doppler formula, namely,

$$
\omega=-\eta_{\mu \nu} k_{0}^{\mu} u^{v}=\gamma\left(\omega_{0}-\mathbf{v} \cdot \mathbf{k}_{0}\right)
$$

Throughout this paper, we use the convention that $c=1$, unless specified otherwise; moreover, Greek indices run from 0 to 3, while Latin indices run from 1 to 3. The Minkowski metric tensor $\eta_{\mu \nu}$ is given by diag $(-1,1,1,1)$.

Let us note that Equation (1) can be expressed as $-\omega_{0} d t+\mathbf{k}_{0} \cdot d \mathbf{x}=-\omega d \tau$, which follows from the invariance of the phase of the wave under Lorentz transformation. Indeed, if we consider the class of moving inertial observers all at rest in the inertial frame of $\mathcal{O}$, we recover from the Lorentz invariance of the phase the relativistic relations for both the frequency $\omega$ and the corresponding wave vector $\mathbf{k}$, namely,

$$
\mathbf{k}=\mathbf{k}_{0}+\frac{1}{v^{2}}(\gamma-1)\left(\mathbf{v} \cdot \mathbf{k}_{0}\right) \mathbf{v}-\gamma \mathbf{v} \omega_{0}
$$

where $v=|\mathbf{v}|$.

The Lorentz invariance of the phase is a consequence of a more basic approach that deals with the electromagnetic radiation field in the two Lorentz frames under consideration here. Transforming the radiation field, either the gauge field $A_{\mu}$ or the field tensor $F_{\mu \nu}=\partial_{\mu} A_{\nu}-\partial_{\nu} A_{\mu}$, from the background 
inertial frame to the moving inertial frame results in the invariance of the radiation's phase under Lorentz transformation.

We now wish to extend our treatment to accelerated observers, since actual observers are all more or less accelerated. Let us for the moment extend the theory of relativity to such observers in the standard manner by applying Lorentz transformations point by point along their accelerated world lines [1]. The pointwise extension of Lorentz invariance is the content of the hypothesis of locality that is at the foundation of the special and general theories of relativity. The locality assumption implies that an accelerated observer is, at each event along its world line, locally equivalent to an otherwise identical momentarily comoving inertial observer. To avoid unphysical situations when dealing with accelerated motion, we generally assume that the acceleration is turned on at some initial time $t_{i}$ and, after a finite interval of time, the acceleration is turned off at $t_{f}$. Moreover, for $t<t_{i}$ and $t>t_{f}$, the observer moves uniformly in an inertial frame of reference. In most laboratory experiments, the accelerations are small and the locality postulate is a good approximation; however, in situations involving high accelerations the locality assumption may not be adequate [2-6].

By way of illustration, let us consider a thought experiment involving an observer that for $t \geq 0$ rotates uniformly about the $z$ axis in the $(x, y)$ plane of an inertial frame of reference. More specifically, for $-\infty<t<0$, the observer under consideration moves uniformly along a straight line parallel to the $y$ axis with speed $r \Omega_{0}$ in the $(x, y)$ plane. That is, $x=r>0$, and $y=r \Omega_{0} t$. At $t=0$, the observer is forced to move counterclockwise with uniform angular velocity $\Omega_{0}>0$ on a circular orbit of radius $r$ about the $z$ axis. Thus, for $t \geq 0, x=r \cos \varphi$ and $y=r \sin \varphi$, where $\varphi=\Omega_{0} t$. The rotating observer with proper time $\tau=t / \gamma$ measures the frequency of a plane monochromatic electromagnetic wave that propagates along the $z$ axis. In this case of normal incidence, the locality postulate leads to the local invariance of the phase and we find from Equation (1),

$$
\omega_{\mathrm{D}}=\gamma \omega_{0}, \quad \gamma=\left(1-v^{2}\right)^{-1 / 2}, \quad v=r \Omega_{0},
$$

which is the expression for the transverse Doppler effect. This result can be physically interpreted in terms of the phenomenon of time dilation. On the other hand, transforming the radiation field to the rotating frame results in Fourier components with frequencies:

$$
\tilde{\omega}_{ \pm}=\gamma\left(\omega_{0} \mp \Omega_{0}\right) .
$$

The actual calculation involved in this more general result can be carried out using the explicit transformation of either $A_{\mu}$ or $F_{\mu \nu}$ to the rotating frame [7] or by means of projecting these fields on the natural orthonormal tetrad frame of the rotating observer [8]. An observer generally carries an adapted orthonormal tetrad frame field $\lambda^{\mu} \hat{\alpha}$ such that:

$$
\eta_{\mu v} \lambda_{\hat{\alpha}}^{\mu} \lambda_{\hat{\beta}}^{v}=\eta_{\hat{\alpha} \hat{\beta}}, \quad \lambda_{\hat{0}}^{\mu}=\frac{d x^{\mu}}{d \tau} .
$$

For $t \geq 0$, the components of the rotating observer's tetrad frame in $(t, x, y, z)$ coordinates are given by:

$$
\begin{aligned}
& \lambda_{\hat{0}}^{\mu}=\gamma(1,-v \sin \varphi, v \cos \varphi, 0), \\
& \lambda_{\hat{1}}^{\mu}=(0, \cos \varphi, \sin \varphi, 0), \\
& \lambda_{\hat{2}}^{\mu}=\gamma(v,-\sin \varphi, \cos \varphi, 0), \\
& \lambda_{\hat{3}}^{\mu}=(0,0,0,1) .
\end{aligned}
$$

Let us note that the unit axes of the observer's spatial frame $\lambda^{\mu}{ }_{\hat{i}}$, for $i=1,2,3$, point in the radial, tangential, and $z$ directions, respectively, in the cylindrical system of coordinates in the background inertial frame. 
The derivation of Equation (4) is based on the application of the hypothesis of locality to the radiation field together with the nonlocal operation of Fourier analysis in time. Indeed, the measured frequency $\tilde{\omega}_{ \pm}$approaches the local Doppler result $\omega_{\mathrm{D}}$ as $\Omega_{0} / \omega_{0} \rightarrow 0$, since:

$$
\tilde{\omega}_{ \pm}=\omega_{\mathrm{D}}\left(1 \mp \frac{\Omega_{0}}{\omega_{0}}\right)
$$

Let us note that $\Omega_{0} / \omega_{0}$ is equal to the ratio of the reduced wavelength of the incident radiation to $c / \Omega_{0}$, which is the acceleration length of the rotating observer. Therefore, it follows from Equation (10) that we recover the pointwise transverse Doppler result in the eikonal (or WKB) limit, moreover, phase invariance in inertial frames does not in general extend to accelerated systems.

Ignoring time dilation for the moment, $\tilde{\omega}_{ \pm} \approx \omega_{0} \mp \Omega_{0}$ comes about as a consequence of the coupling of the helicity of the radiation with the rotation of the observer. The incident radiation can be expressed in the circular polarization basis as a linear superposition of positive and negative helicity components. In the positive (negative) helicity case, static inertial observers in the background global inertial frame observe that the electric and magnetic radiation fields rotate in the positive (negative) sense with frequency $\omega_{0}$ about the direction of the wave's propagation. From the viewpoint of the rotating observer, the electric and magnetic radiation fields rotate with frequency $\omega_{0}-\Omega_{0}\left(\omega_{0}+\Omega_{0}\right)$ in the positive (negative) helicity case. This "angular Doppler effect" has been studied in classical optics, see [9] and the references cited therein. The helicity-rotation coupling is responsible for the phenomenon of phase wrap-up in the GPS [10]. It is also the origin of a certain frequency shift that occurs when circularly polarized radiation passes through a rotating spin flipper. Indeed, for $\Omega_{0} \ll \omega_{0}$, this phenomenon has been observationally known for a long time [11,12].

Imagine a uniformly rotating half-wave plate (HWP) instead of the rotating observer in our thought experiment. At the lower edge of the plate the frequency of an incident positive-helicity wave is $\omega^{\prime} \approx \omega_{0}-\Omega_{0}$. Inside the plate, the frequency remains constant and equal to $\omega^{\prime}$ as the rotation of the plate is assumed to be uniform. At the upper edge of the plate, the connection of $\omega^{\prime}$ with the frequency $\omega_{1}$ of the emerging negative-helicity wave is $\omega^{\prime} \approx \omega_{1}+\Omega_{0}$, so that $\omega_{1}-\omega_{0} \approx-2 \Omega_{0}$. There is therefore a downshift in the frequency of the radiation as it passes through the spin flipper. A corresponding upshift would occur for incident negative-helicity radiation. The general formula for the energy shift is $\mp 2 \hbar s \Omega_{0}$, where $s$ is the spin of the radiation field [13]. Further discussion of helicity-rotation coupling for photons is contained in [8,14-17]. The spin-rotation coupling is a general phenomenon that is a manifestation of the inertia of intrinsic spin and has extensive observational support and in fact, it has recently been observed directly in neutron interferometry [18-45].

Let us now return to Equation (4) and note that this equation has observational support only for $\Omega_{0} \ll \omega_{0}$. Are there higher-order terms in Equation (4)? For instance, could there be terms of order $\left(\Omega_{0} / \omega_{0}\right)^{2}$ or higher in wave expansion (10)? The existence of such terms is quite unlikely; otherwise, there would be divergence as $\omega_{0} \rightarrow 0$. Various aspects of such problems have been discussed in [7]. There is, however, one important issue that we need to address, namely, the possibility that for incident positive-helicity radiation of frequency $\omega_{0}=\Omega_{0}$, we have $\tilde{\omega}_{+}=0$. By a mere rotation of angular velocity $\Omega_{0}=\omega_{0}$, the whole radiation field stands completely still with respect to the rotating observer. This is not possible for inertial observers in Minkowski spacetime, since $\omega=0$ implies $\omega_{0}=0$ in Equation (1). However, before the advent of special relativity theory, an observer could in principle move at the speed of light and hence stay completely at rest with a radiation field. The situation here is therefore reminiscent of the pre-relativistic Doppler effect and indeed, Einstein mentioned such a puzzling possibility in connection with the pre-relativistic Doppler formula in his autobiographical notes [46]. This conceptual difficulty was removed for inertial observers via the Lorentz invariance of physical laws. We do the same via an appropriate nonlocal extension of the locality hypothesis of the standard special relativity theory.

The hypothesis of locality, as applied to the electromagnetic radiation field, is the origin of the circumstance that a radiation field can in principle stand completely still with respect to a rotating 
observer. The locality postulate in turn originates from classical mechanics where the state of a classical particle is determined by its position and velocity. On the other hand, in accordance with the Huygens principle, wave phenomena are in general nonlocal. In 1993, a nonlocal theory of accelerated systems was put forward in which the past history of accelerated observers was taken into account via kernels that would be constructed so as to mitigate the conceptual difficulty of radiation standing completely still [47]. Using electrons in electromagnetic fields as accelerated systems in the correspondence limit, we have shown that the nonlocal theory is in better agreement with quantum mechanics than the standard theory based on the locality postulate [48]. We now turn to a brief description of the theory of acceleration-induced nonlocality, which leads to nonlocal special relativity theory [49,50].

\section{Nonlocality of Accelerated Systems}

Imagine an accelerated observer following a path $x^{\mu}(\tau)$ in Minkowski spacetime in the presence of a field $\psi(x)$, where $x$ here represents an event $x^{\mu}$. What is the field that the accelerated observer measures? According to the locality postulate of the standard relativity theory, Lorentz invariance can be applied point by point along the path and in this way, the observer measures a field:

$$
\tilde{\psi}(\tau)=\Lambda(\tau) \psi(x(\tau))
$$

where $\Lambda$ is a matrix representation of the Lorentz group. On the other hand, according to Bohr and Rosenfeld, it is not possible to measure even the classical electromagnetic field at a spacetime event by inertial observers. Indeed, a spacetime averaging procedure is necessarily involved in such a measurement process [51,52].

Following Bohr and Rosenfeld [51], consider for the sake of illustration an extended body of volume $V$ and uniform charge density $\bar{\rho}$ that is placed in an external electric field $\mathbf{E}(t, \mathbf{x})$. The Lorentz force law is indispensable for the measurement of the electromagnetic field; indeed, the electric and magnetic fields are defined via the Lorentz force law. In this case, we have:

$$
\frac{d \mathbf{P}}{d t}=\bar{\rho} \int_{V} \mathbf{E}(t, \mathbf{x}) d^{3} x
$$

Measuring the momentum of the extended charged body $\mathbf{P}$ at $t^{\prime}$ and $t^{\prime \prime}>t^{\prime}$, we get:

$$
\mathbf{P}\left(t^{\prime \prime}\right)-\mathbf{P}\left(t^{\prime}\right)=\bar{\rho} \int_{t^{\prime}}^{t^{\prime \prime}} \int_{V} \mathbf{E}(t, \mathbf{x}) d^{3} x
$$

thus, the field as measured by static inertial observers is given by:

$$
<\mathbf{E}>=\frac{1}{\Delta} \int_{\Delta} \mathbf{E}(x) d^{4} x
$$

where $\Delta=\left(t^{\prime \prime}-t^{\prime}\right) V$.

Extending the Bohr-Rosenfeld argument to accelerated systems is a daunting task. Instead, we implement the averaging requirement as follows: Let $\Psi(\tau)$ be the field actually measured by the accelerated observer; then, the most general linear relationship linking $\Psi(\tau)$ to $\tilde{\psi}(\tau)=\Lambda(\tau) \psi(x(\tau))$ that is consistent with causality is given by:

$$
\Psi(\tau)=\tilde{\psi}(\tau)+\int_{\tau_{0}}^{\tau} \mathcal{K}\left(\tau, \tau^{\prime}\right) \tilde{\psi}\left(\tau^{\prime}\right) d \tau^{\prime}
$$

for $\tau \geq \tau_{0}$, where $\tau_{0}$ is the instant at which the acceleration is turned on. In this ansatz, which is consistent with the superposition principle, the locality postulate is extended by a certain average over the past world line of the observer. 
The Volterra integral equation of the second kind (15) relates $\psi$ to $\Psi$ via $\tilde{\psi}$ given by Equation (11) and the resulting connection is unique in the space of continuous functions by Volterra's theorem, which has been extended to the space of square-integrable functions by Tricomi [53,54]. The Volterra-Tricomi uniqueness result is an important feature of the nonlocal theory of accelerated systems.

The kernel of ansatz (15), $\mathcal{K}\left(\tau, \tau^{\prime}\right)$, is expected to vanish in the absence of acceleration. Moreover, the kernel should be such that a basic radiation field could never stand completely still with respect to an accelerated observer. Detailed investigations have revealed that a consistent theory can be developed based on:

$$
\mathcal{K}\left(\tau, \tau^{\prime}\right)=K\left(\tau^{\prime}\right)=-\frac{d \Lambda\left(\tau^{\prime}\right)}{d \tau^{\prime}} \Lambda^{-1}\left(\tau^{\prime}\right)
$$

see [49] and the references cited therein. The formula for $K(\tau)$, which vanishes in the absence of the acceleration of the observer, simply follows from the assumption that a constant field $\psi$ will lead to a constant $\Psi$ in ansatz (15). The Volterra-Tricomi uniqueness theorem then implies that an actual radiation field will never be constant as measured by accelerated observers. Moreover, after the acceleration has been turned off at $\tau_{f}$, the memory of past acceleration remains as a constant addition to the measured field; that is, for $\tau \geq \tau_{f}$,

$$
\Psi(\tau)=\tilde{\psi}(\tau)+\int_{\tau_{0}}^{\tau_{f}} K\left(\tau^{\prime}\right) \tilde{\psi}\left(\tau^{\prime}\right) d \tau^{\prime} .
$$

The nonlocal theory of accelerated systems has been applied to the Dirac field with satisfactory results [55]. There is a problem, however, in the case of the electromagnetic field $F_{\mu \nu}$ due to the existence of electrostatic and magnetostatic fields. To see this, let us note that with the substitution of kernel (16), ansatz (15) can be written as:

$$
\Psi(\tau)=\tilde{\psi}\left(\tau_{0}\right)+\int_{\tau_{0}}^{\tau} \Lambda\left(\tau^{\prime}\right) \frac{d \psi\left(\tau^{\prime}\right)}{d \tau^{\prime}} d \tau^{\prime}
$$

It follows that in a static background electromagnetic field, an accelerated observer will always measure a constant field $\tilde{\psi}\left(\tau_{0}\right)$, which contradicts experimental results involving small accelerations that appear to be in general agreement with the locality postulate of the special relativity theory [56-58]. To resolve this difficulty, one can assume that kernel $K$ given by Equation (16) is appropriate for the vector potential $A_{\mu}$, while a different kernel should be chosen for the field tensor $F_{\mu \nu}$. A specific choice for such a kernel involving parity violation has been put forward in [59]. The purpose of the present paper is to re-examine our previous proposal and provide a deeper and more general analysis of the problem.

\section{Nonlocal Electrodynamics}

According to the hypothesis of locality, the vector potential $A_{\mu}$ and the field tensor $F_{\mu v}$ as measured by the accelerated observer are:

$$
\tilde{A}_{\hat{\alpha}}=A_{\mu} \lambda_{\hat{\alpha}}^{\mu}, \quad \tilde{F}_{\hat{\alpha} \hat{\beta}}=F_{\mu \nu} \lambda_{\hat{\alpha}}^{\mu} \lambda_{\hat{\beta}}^{v}
$$

On the other hand, we claim that the actually measured fields after the acceleration is turned on at $\tau_{0}$ are:

$$
\begin{gathered}
\mathcal{A}_{\hat{\alpha}}(\tau)=\tilde{A}_{\hat{\alpha}}(\tau)+\int_{\tau_{0}}^{\tau} K_{\hat{\alpha}}^{\hat{\beta}}\left(\tau^{\prime}\right) \tilde{A}_{\hat{\beta}}\left(\tau^{\prime}\right) d \tau^{\prime}, \\
\mathcal{F}^{\hat{\alpha} \hat{\beta}}(\tau)=\tilde{F}^{\hat{\alpha} \hat{\beta}}(\tau)+\int_{\tau_{0}}^{\tau} K_{\hat{\gamma} \hat{\alpha}}^{\hat{\alpha} \hat{\beta}}\left(\tau^{\prime}\right) \tilde{F}^{\hat{\gamma} \hat{\delta}}\left(\tau^{\prime}\right) d \tau^{\prime} .
\end{gathered}
$$

Once the kernels are fixed, other aspects of the nonlocal electrodynamics of accelerated systems can be determined. For the kernel of Equation (20) involving the vector potential $A_{\mu}$, the simplest possibility would be to choose the kernel given by Equation (16). If $A_{\mu}$ is constant, then $F_{\mu \nu}=0$ 
everywhere and via Equation (18) we have that $\mathcal{A}_{\hat{\alpha}}(\tau)=\tilde{A}_{\hat{\alpha}}\left(\tau_{0}\right)$ is constant as well. This circumstance does not appear to pose any difficulty and it is clear that under the gauge transformation $A_{\mu} \mapsto$ $A_{\mu}+\partial_{\mu} \Sigma, \tilde{A}_{\hat{\alpha}} \mapsto \tilde{A}_{\hat{\alpha}}+\left(\partial_{\mu} \Sigma\right) \lambda_{\hat{\alpha}}^{\mu}$ and, similarly, $\mathcal{A}_{\hat{\alpha}}$ will be subject to a nonlocal gauge transformation, but Equation (21) will remain invariant.

The kernels depend upon the acceleration of the observer; therefore, a description of the acceleration tensor is required.

\subsection{Acceleration Tensor}

Along the observer's world line, the motion of the adapted tetrad frame can be expressed as:

$$
\frac{d \lambda_{\hat{\alpha}}^{\mu}}{d \tau}=\phi_{\hat{\alpha}}^{\hat{\beta}} \lambda_{\hat{\beta}}^{\mu}, \quad \phi_{\hat{\alpha} \hat{\beta}}=-\phi_{\hat{\beta} \hat{\alpha}} .
$$

The antisymmetry of the acceleration tensor $\phi_{\hat{\alpha} \hat{\beta}}$ is a simple consequence of the orthonormality of the adapted tetrad frame.

In general, the acceleration tensor has a decomposition, $\phi_{\hat{\alpha} \hat{\beta}} \mapsto(-\mathbf{g}, \Omega)$, in close analogy with the electromagnetic field tensor, namely, $F_{\mu v} \mapsto(\mathbf{E}, \mathbf{B})$, where $F_{0 i}=-E_{i}$ and $F_{i j}=\epsilon_{i j k} B^{k}$ in our convention. The spacetime invariants $\mathbf{g}$ and $\Omega$ have local components with respect to the adapted frame. The "electric" part of the acceleration tensor corresponds to the translational acceleration of the observer, $g_{\hat{i}}=\phi_{\hat{0} \hat{i}}$, while the "magnetic" part, $\Omega_{\hat{i}}=\frac{1}{2} \epsilon_{\hat{i} \hat{j} \hat{k}} \phi^{\hat{j} \hat{k}}$, corresponds to the angular velocity of the spatial frame of the observer relative to a nonrotating frame that is Fermi-Walker transported along the observer's world line.

\subsection{Acceleration Kernels}

It follows from inspection of Equations (11) and (16) as well as (19) and (22) that for the kernel of the vector potential we have:

$$
K_{\hat{\alpha}}^{\hat{\beta}}=-\phi_{\hat{\alpha}}^{\hat{\beta}}
$$

which we must use in Equation (20).

Let us next turn to the evaluation of the field kernel in Equation (21), $K^{\hat{\alpha} \hat{\beta}} \hat{\gamma} \hat{\delta}$, which is antisymmetric in its first and second pairs of indices and is the main subject of the present paper. It should be constructed out of the Minkowski metric tensor $\eta_{\hat{\alpha} \hat{\beta}}$, the Levi-Civita tensor $\epsilon_{\hat{\alpha} \hat{\beta} \hat{\gamma} \hat{\delta}}$ with $\epsilon_{\hat{0} \hat{1} \hat{z} \hat{3}}=1$, and the acceleration tensor $\phi_{\hat{\alpha} \hat{\beta}}$. In this connection, the field kernel corresponding to Equation (16) is given by:

$$
\kappa_{\hat{\alpha} \hat{\beta} \hat{\gamma} \hat{\delta}}=-\frac{1}{2}\left(\phi_{\hat{\alpha} \hat{\gamma}} \eta_{\hat{\beta} \hat{\delta}}+\phi_{\hat{\beta} \hat{\delta}} \eta_{\hat{\alpha} \hat{\gamma}}-\phi_{\hat{\beta} \hat{\gamma}} \eta_{\hat{\alpha} \hat{\delta}}-\phi_{\hat{\alpha} \hat{\delta}} \eta_{\hat{\beta} \hat{\gamma}}\right)
$$

which, as explained before, cannot be the electromagnetic field kernel by itself. Indeed, in previous work [59], we assumed:

$$
K_{\hat{\alpha} \hat{\beta} \hat{\gamma} \hat{\delta}}=p \kappa_{\hat{\alpha} \hat{\beta} \hat{\gamma} \hat{\delta}}+q^{*} \kappa_{\hat{\alpha} \hat{\beta} \hat{\gamma} \hat{\delta}}
$$

where $p$ and $q$ are constant real dimensionless parameters and ${ }^{*} \kappa$ is the dual of $\kappa$,

$$
{ }^{*} \kappa^{\hat{\alpha} \hat{\beta}} \hat{\gamma} \hat{\delta}=\frac{1}{2} \epsilon_{\hat{\rho} \hat{\alpha}}^{\hat{\alpha}} \kappa_{\hat{\rho} \hat{\sigma} \hat{\delta}}^{\hat{\sigma}} .
$$

The right and left duals of $\kappa$ turn out to be equal as a consequence of the antisymmetry of the acceleration tensor. Clearly, the existence of $q \neq 0$ violates parity conservation. On the other hand, the adoption of kernel (25) was based on the additional assumption that $K$ is linearly dependent upon the acceleration tensor $\phi_{\hat{\alpha} \hat{\beta}}$.

Let us observe that acceleration-induced nonlocal electrodynamics shares similarities with the nonlocal electrodynamics of media [60,61]. Even in the linear electrodynamics of media, there is evidence for parity violation, see [62-65] and the references cited therein. However, no corresponding observational evidence regarding accelerated systems is available at present. The main purpose of 
the present work is to point out that one can remove the linearity constraint on the field kernel and thereby develop an acceleration-induced nonlocal electrodynamics that preserves parity conservation. The mathematical form of the various possible field kernels are explored in the last part of Section 4 .

Specifically, in the present work we assume a field kernel that is quadratic in the acceleration tensor and is of the form:

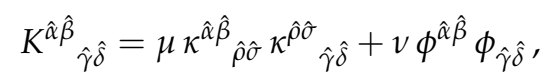

where $\mu$ and $v$ are constant real parameters that carry dimensions of length. The new field kernel is symmetric; that is, $K_{\hat{\alpha} \hat{\beta} \hat{\gamma} \hat{\delta}}=K_{\hat{\gamma} \hat{\delta} \hat{\alpha} \hat{\beta}}$. In this paper, we work out the physical consequences of kernel (27) for the sake of illustration. In principle, a parity conserving kernel could be a sum of linear, quadratic, and higher-order terms in the acceleration tensor.

\section{Parity Violation}

Soon after the discovery of parity violation in weak interactions, Stueckelberg pointed out the theoretical possibility of parity violation in gravity due to the existence of the covariantly constant Levi-Civita totally antisymmetric tensor field $\epsilon_{\mu \nu \rho \sigma}$ in general relativity [66]. Indeed, this tensor leads to the definition of dual fields that could help induce parity violation. An instance of this circumstance is given in Equation (25), the kernel of previously published nonlocal electrodynamics of accelerated systems $[6,49,59]$. Observational consequences of the possible breakdown of parity and time-reversal invariance in the gravitational interaction have been considered by a number of authors, see for instance [67-77] and the references cited therein. On the other hand, there is no parity violation in standard general relativity. Furthermore, parity conservation is maintained in the standard electrodynamics of accelerated systems and gravitational fields, see Appendix A for a detailed discussion.

In acceleration-induced nonlocal electrodynamics, when one assumes that the kernel is only linear in acceleration, the existence of parity violating term in Equation (25) becomes absolutely necessary; otherwise, there would be inconsistency in the physical results of the theory. A somewhat similar situation occurs in the gravitational context involving the torsion pseudovector in the constitutive relation of nonlocal gravity $[6,78,79]$. A brief discussion of parity violation in nonlocal gravity is contained in Section 8.

There is no observational data regarding accelerated systems that could be used to exclude completely parity violation in electrodynamics. The main purpose of the present paper is to examine quadratic terms in acceleration that could supplant parity violating terms in the kernel. The rest of this paper is devoted to the derivation of some consequences of parity conserving nonlocal electrodynamics of accelerated systems. To this end, it is convenient to express the main integral relation (21) in matrix form.

\section{Matrix Representation}

For the sake of simplicity, we wish to represent our main field ansatz as a matrix equation. We use the antisymmetry of the field tensor $F^{\mu v}$ and assume that its pair of indices in effect takes the values $\{01,02,03,23,31,12\}$ and we can then take care of the remaining possibilities by means of a simple factor of 2 . Thus, we replace $F^{\mu v}$ by a column 6-vector $F$ with components $\mathbf{E}$ and $\mathbf{B}$, since $E_{i}=F^{0 i}$ and $B_{i}=\frac{1}{2} \epsilon_{i j k} F^{j k}$ in our convention,

$$
F=\left[\begin{array}{l}
\mathbf{E} \\
\mathbf{B}
\end{array}\right] \text {. }
$$

Similarly, we replace kernel $K^{\hat{\alpha} \hat{\beta}} \hat{\gamma} \hat{\delta}$ by a $6 \times 6$ matrix $\mathbb{K}$, where the first and second pair of indices range over the set $\{01,02,03,23,31,12\}$. Equation (21) then takes the form:

$$
\mathcal{F}(\tau)=\tilde{F}(\tau)+2 \int_{\tau_{0}}^{\tau} \mathbb{K}\left(\tau^{\prime}\right) \tilde{F}\left(\tau^{\prime}\right) d \tau^{\prime}
$$


where $\tilde{F}=\Lambda F$ and $\Lambda$ is a $6 \times 6$ matrix that can be determined from the adapted tetrad frame field.

\section{Relations Involving Kernels}

We collect here a number of relations that will be useful in explicit applications. It follows from Equation (24) that for the special kernel $\kappa$ we have:

$$
\kappa_{\hat{\alpha} \hat{\beta} \hat{\gamma} \hat{\delta}}=-\kappa_{\hat{\gamma} \hat{\delta} \hat{\alpha} \hat{\beta}}, \quad \kappa_{\hat{\alpha} \hat{\alpha} \hat{\beta}}^{\mu}=-\phi_{\hat{\alpha} \hat{\beta}}, \quad \kappa_{\hat{\alpha} \hat{\beta}}^{\hat{\alpha} \hat{\beta}}=0 .
$$

Moreover, ${ }^{*} \kappa=\kappa^{*}$ with:

$$
\kappa_{\hat{\alpha} \hat{\beta}}^{*} \hat{\gamma} \hat{\delta}=\frac{1}{2} \kappa_{\hat{\alpha} \hat{\beta}}^{\hat{\rho} \hat{\sigma}} \epsilon_{\hat{\rho} \hat{\sigma}}^{\hat{\gamma} \hat{\delta}}=-\frac{1}{2}\left(\phi_{\hat{\alpha}}^{\hat{\rho}} \epsilon_{\hat{\rho} \hat{\beta}}^{\hat{\gamma} \hat{\delta}}-\phi_{\hat{\beta}}^{\hat{\rho}} \epsilon_{\hat{\rho} \hat{\alpha}}^{\hat{\gamma} \hat{\delta}}\right) .
$$

The mixed dual:

$$
\zeta_{\hat{\alpha} \hat{\beta}}^{\hat{\gamma} \hat{\delta}}=\frac{1}{2} \kappa_{\hat{\alpha} \hat{\rho} \hat{\sigma} \hat{\beta}} \epsilon^{\hat{\rho} \hat{\sigma} \hat{\gamma} \hat{\delta}}
$$

is symmetric in its first pair of indices:

$$
\zeta_{\hat{\alpha} \hat{\beta}}^{\hat{\gamma} \hat{\delta}}=\frac{1}{4}\left(\kappa_{\hat{\alpha} \hat{\rho} \hat{\sigma} \hat{\beta}}-\kappa_{\hat{\alpha} \hat{\sigma} \hat{\rho} \hat{\beta}}\right) \epsilon^{\hat{\rho} \hat{\sigma} \hat{\sigma} \hat{\delta}}=\frac{1}{4}\left(\kappa_{\hat{\alpha} \hat{\rho} \hat{\sigma} \hat{\beta}}+\kappa_{\hat{\beta} \hat{\rho} \hat{\sigma} \hat{\alpha}}\right) \epsilon^{\hat{\rho} \hat{\sigma} \hat{\sigma} \hat{\delta}} .
$$

It is therefore not suitable as a field kernel in any case; here, we correct the erroneous assertion that the mixed dual vanishes [49,59].

In expression (24) for the special kernel $\kappa$, we can replace the acceleration tensor $\phi_{\hat{\alpha} \hat{\beta}}$ by its dual $\phi_{\hat{\alpha} \hat{\beta}}^{*}$ and thereby obtain $\chi_{\hat{\alpha} \hat{\beta} \hat{\gamma} \hat{\delta}}$, then,

$$
\chi_{\hat{\alpha} \hat{\beta}}^{\hat{\gamma} \hat{\delta}}=\kappa_{\hat{\alpha} \hat{\beta}}^{*} \hat{\gamma} \hat{\delta}, \quad \chi_{\hat{\alpha} \hat{\beta}}^{*} \hat{\hat{\delta}} \hat{\delta}=-\kappa_{\hat{\alpha} \hat{\beta}}^{\hat{\gamma} \hat{\delta}},
$$

so that the double duality operation equals minus the identity operation in this case.

Regarding quadratic terms in the acceleration tensor, we recall that:

$$
\phi_{\hat{\alpha} \hat{\beta}} \phi^{\hat{\alpha} \hat{\beta}}=-2\left(g^{2}-\Omega^{2}\right), \quad \phi_{\hat{\alpha} \hat{\beta}}^{*} \phi^{\hat{\alpha} \hat{\beta}}=-2 \mathbf{g} \cdot \Omega .
$$

Using a function $W$ of these invariants, we can form field kernels of the form $W \epsilon^{\hat{\alpha} \hat{\beta}} \hat{\gamma}$. Moreover, we find:

$$
\kappa^{\hat{\alpha} \hat{\beta}}{ }_{\hat{\gamma} \hat{\delta}} \kappa^{\hat{\gamma} \hat{\delta}_{\hat{\rho}} \hat{\sigma}}=\phi_{\hat{\rho}}^{\hat{\alpha}} \phi_{\hat{\beta}}^{\hat{\beta}}-\phi^{\hat{\alpha}} \hat{\sigma} \phi^{\hat{\beta}}{ }_{\hat{\rho}}+\frac{1}{2}\left(\phi^{\hat{\alpha}}{ }_{\hat{\gamma}} \phi_{\hat{\gamma}}^{\hat{\gamma}} \delta_{\hat{\sigma}}^{\hat{\beta}}-\phi^{\hat{\alpha}}{ }_{\hat{\gamma}} \phi^{\hat{\gamma}} \hat{\sigma} \delta_{\hat{\rho}}^{\hat{\beta}}-\phi^{\hat{\beta}} \hat{\gamma} \phi^{\hat{\gamma}} \hat{\rho} \delta_{\hat{\sigma}}^{\hat{\alpha}}+\phi^{\hat{\beta}}{ }_{\hat{\gamma}} \phi^{\hat{\gamma}} \delta_{\hat{\sigma}}^{\hat{\alpha}}\right)
$$

and

$$
\kappa_{\hat{\alpha} \hat{\alpha} \hat{\delta}}^{\hat{\beta}} \kappa_{\hat{\alpha} \hat{\beta}}^{\hat{\beta}}=-2 \phi_{\hat{\rho} \hat{\sigma}} \phi^{\hat{\rho} \hat{\sigma}} .
$$

In connection with the matrix representation, a detailed treatment reveals that for the special kernel $\kappa$ we have:

$$
\kappa_{\hat{\gamma} \hat{\alpha} \hat{\delta}}^{\hat{\alpha} \hat{\beta}} \mapsto \kappa=\frac{1}{2}\left[\begin{array}{cc}
\kappa_{1} & -\kappa_{2} \\
\kappa_{2} & \kappa_{1}
\end{array}\right],
$$

where the $3 \times 3$ matrices $\kappa_{1}$ and $\kappa_{2}$ are given by:

$$
\kappa_{1}=\mathbf{\Omega} \cdot \mathbf{I}, \quad \kappa_{2}=\mathbf{g} \cdot \mathbf{I} .
$$

Here, $I_{i}$ is proportional to the operator of infinitesimal rotations about the $x^{i}$ axis, so that $I_{1}, I_{2}$, and $I_{3}$ form a basis for the Lie algebra of the rotation group as a 3-dimensional vector space, that is,

$$
\left(I_{i}\right)_{j k}=-\epsilon_{i j k}, \quad\left[I_{i}, I_{j}\right]=\epsilon_{i j k} I_{k} .
$$


Let us note that in our matrix representation:

$$
\epsilon_{\hat{\gamma} \hat{\delta}}^{\hat{\alpha}} \mapsto\left[\begin{array}{cc}
0 & -I \\
I & 0
\end{array}\right]
$$

where $I=\operatorname{diag}(1,1,1)$ is the identity matrix; therefore, Equation (26) results in:

$$
{ }^{*}{ }^{\hat{\alpha} \hat{\beta}}{ }_{\hat{\gamma} \hat{\delta}} \mapsto{ }^{*} \kappa=\frac{1}{2}\left[\begin{array}{cc}
-\kappa_{2} & -\kappa_{1} \\
\kappa_{1} & -\kappa_{2}
\end{array}\right] .
$$

Using the matrix representation, it is straightforward to show that:

$$
\kappa^{\hat{\alpha} \hat{\beta}} \hat{\rho} \hat{\sigma} \kappa^{\hat{\rho} \hat{\sigma}} \hat{\gamma} \hat{\delta}=-{ }^{*} \kappa^{\hat{\alpha} \hat{\beta}}{ }_{\hat{\rho} \sigma}{ }^{*} \kappa^{\hat{\rho} \hat{\sigma}}{ }_{\hat{\gamma} \hat{\delta}}, \quad * \kappa^{\hat{\alpha} \hat{\beta}}{ }_{\hat{\rho} \hat{\sigma}} \kappa^{\hat{\rho} \hat{\sigma}}{ }_{\hat{\gamma} \hat{\delta}}=\kappa^{\hat{\alpha} \hat{\beta}}{ }_{\hat{\rho} \hat{\sigma}}{ }^{*} \kappa^{\hat{\rho} \hat{\sigma}} \hat{\gamma} \hat{\delta} .
$$

Furthermore,

$$
\kappa^{\hat{\alpha} \hat{\beta}} \hat{\rho} \hat{\sigma} \kappa^{\hat{\rho} \hat{\sigma}} \hat{\gamma} \hat{\delta} \mapsto \frac{1}{2}\left[\begin{array}{cc}
\kappa_{1}^{2}-\kappa_{2}^{2} & -\left(\kappa_{1} \kappa_{2}+\kappa_{2} \kappa_{1}\right) \\
\kappa_{1} \kappa_{2}+\kappa_{2} \kappa_{1} & \kappa_{1}^{2}-\kappa_{2}^{2}
\end{array}\right],
$$

where

$$
\operatorname{tr}\left(\kappa_{1}^{2}\right)=-2 \Omega^{2}, \quad \operatorname{tr}\left(\kappa_{2}^{2}\right)=-2 g^{2} .
$$

For the other quadratic kernel in Equation (27), we find the $6 \times 6$ representation:

$$
\phi^{\hat{\alpha} \hat{\beta}} \phi_{\hat{\gamma} \hat{\delta}} \mapsto\left[\begin{array}{cc}
-g_{i} g_{j} & -g_{i} \Omega_{j} \\
\Omega_{i} g_{j} & \Omega_{i} \Omega_{j}
\end{array}\right] ;
$$

indeed, similar expressions exist for ${ }^{*} \phi^{\hat{\alpha} \hat{\beta}} \phi_{\hat{\gamma} \hat{\delta}}$ as well as $\phi^{\hat{\alpha} \hat{\beta} *} \phi_{\hat{\gamma} \hat{\delta}}$.

Assuming parity conservation, our quadratic kernel $\mathbb{K}$ given by Equation (27) has a matrix representation that is given by $\mu$ times the matrix in Equation (44) plus $v$ times the matrix in Equation (46); that is,

$$
\mathbb{K}=\frac{\mu}{2}\left[\begin{array}{cc}
\kappa_{1}^{2}-\kappa_{2}^{2} & -\left(\kappa_{1} \kappa_{2}+\kappa_{2} \kappa_{1}\right) \\
\kappa_{1} \kappa_{2}+\kappa_{2} \kappa_{1} & \kappa_{1}^{2}-\kappa_{2}^{2}
\end{array}\right]+v\left[\begin{array}{cc}
-g_{i} g_{j} & -g_{i} \Omega_{j} \\
\Omega_{i} g_{j} & \Omega_{i} \Omega_{j}
\end{array}\right] .
$$

We examine the physical consequences of the quadratic kernel (27) in the next three sections. In Section 5, we use the matrix formalism to explore helicity-rotation coupling, while in Sections 6 and 7 we study the case of constant background electromagnetic fields. We will find that of the two terms in our proposed quadratic kernel (47), only the first term gives reasonable results, while the second term must vanish (i.e., $v=0$ ). Section 8 contains a general discussion of our results.

\section{Influence of the Quadratic Kernel on Helicity-Rotation Coupling}

The purpose of this section is to examine the implications of kernel (47) for the phenomenon of spin-rotation coupling. We therefore imagine an incident plane monochromatic electromagnetic wave that is normally incident on the rotating observer under consideration in this work. Equation (29) is linear by assumption hence, we can employ complex fields in our calculations and assign physical significance to their real parts in our convention. We represent the incident wave in the circular polarization basis by:

$$
F_{ \pm}(t, \mathbf{x})=i \omega_{0} a_{ \pm}\left[\begin{array}{l}
\mathbf{e}_{ \pm} \\
\mathbf{b}_{ \pm}
\end{array}\right] e^{-i \omega_{0}(t-z)}
$$

where

$$
\mathbf{e}_{ \pm}=\frac{1}{\sqrt{2}}(\widehat{\mathbf{x}} \pm i \widehat{\mathbf{y}}), \quad \mathbf{b}_{ \pm}=\mp i \mathbf{e}_{ \pm}, \quad \mathbf{e}_{ \pm} \cdot \mathbf{e}_{ \pm}^{*}=1
$$


and the upper (lower) sign represents positive (negative) helicity radiation. Here, $\widehat{\mathbf{x}}$ and $\widehat{\mathbf{y}}$ are unit vectors in $x$ and $y$ directions, respectively, while $\omega_{0}$ is the frequency and $a_{+}\left(a_{-}\right)$is the constant amplitude of positive (negative) helicity incident radiation.

Next, we imagine the instantaneous projection of this field on the tetrad frame (6)-(9) of the rotating observer. The acceleration tensor for the rotating observer has a translational part that corresponds to the centripetal acceleration $\mathbf{g}$ and a rotational part that corresponds to the angular velocity $\Omega$ with respect to a locally nonrotating frame. These have components $\mathbf{g}=-v \gamma^{2} \Omega_{0}(1,0,0)$ and $\Omega=\gamma^{2} \Omega_{0}(0,0,1)$ with respect to the local adapted spatial frame $\lambda_{\hat{i}}^{\mu}, i=1,2,3$, that indicate the radial, tangential, and $z$ directions, respectively. The projected field $\tilde{F}$ is given by $\tilde{F}=\Lambda F$, where the $6 \times 6$ matrix $\Lambda$ is obtained from Equation (19) and can be expressed as:

$$
\Lambda=\left[\begin{array}{cc}
\Lambda_{1} & -\Lambda_{2} \\
\Lambda_{2} & \Lambda_{1}
\end{array}\right]
$$

where

$$
\Lambda_{1}=\left[\begin{array}{ccc}
\gamma \cos \varphi & \gamma \sin \varphi & 0 \\
-\sin \varphi & \cos \varphi & 0 \\
0 & 0 & \gamma
\end{array}\right], \quad \Lambda_{2}=v \gamma\left[\begin{array}{ccc}
0 & 0 & -1 \\
0 & 0 & 0 \\
\cos \varphi & \sin \varphi & 0
\end{array}\right]
$$

Let us recall here that $\varphi=\Omega_{0} t=\gamma \Omega_{0} \tau$.

According to the locality postulate, the projected field $\tilde{F}$ measured by the momentarily comoving inertial observers along the world line of the rotating observer is given by:

$$
\tilde{F}_{ \pm}(\tau)=i \gamma \omega_{0} a_{ \pm}\left[\begin{array}{c}
\tilde{\mathbf{e}}_{ \pm} \\
\tilde{\mathbf{b}}_{ \pm}
\end{array}\right] e^{-i \tilde{\omega}_{ \pm} \tau}
$$

where

$$
\tilde{\mathbf{e}}_{ \pm}=\frac{1}{\sqrt{2}}\left[\begin{array}{c}
1 \\
\pm i \gamma^{-1} \\
\pm i v
\end{array}\right], \quad \tilde{\mathbf{b}}_{ \pm}=\mp i \tilde{\mathbf{e}}_{ \pm}, \quad \tilde{\mathbf{e}}_{ \pm} \cdot \tilde{\mathbf{e}}_{ \pm}^{*}=1
$$

As expected, we recover Equation (4), $\tilde{\omega}_{ \pm}=\gamma\left(\omega_{0} \mp \Omega_{0}\right)$, which is the frequency of the wave as measured by the momentarily tangent inertial observer; furthermore, the relative strength of the measured amplitudes of the helicity states in Equation (52) is still $\left(a_{+} / a_{-}\right)$and is therefore not affected by the observer's rotation.

Finally, we must compute the integral of $\mathbb{K} \tilde{F}$ over the proper time of the rotating observer in accordance with Equation (29). The second term in the quadratic kernel (47), which is represented by matrix (46), does not contribute to the integral in Equation (29) because $\phi_{\hat{\alpha} \hat{\beta}} \tilde{F}^{\tilde{\alpha} \hat{\beta}}=0$ in this case based on the circumstance that $g_{1}+v \Omega_{3}=0$ for the uniformly rotating observer. Regarding the first term in Equation (47), we have:

$$
\kappa_{1}^{2}=-\gamma^{4} \Omega_{0}^{2} \operatorname{diag}(1,1,0), \quad \kappa_{2}^{2}=-v^{2} \gamma^{4} \Omega_{0}^{2} \operatorname{diag}(0,1,1) .
$$

Moreover, the elements of matrix (44) are given by:

$$
\kappa_{1}^{2}-\kappa_{2}^{2}=-\gamma^{4} \Omega_{0}^{2} \operatorname{diag}\left(1, \gamma^{-2},-v^{2}\right), \quad \kappa_{1} \kappa_{2}+\kappa_{2} \kappa_{1}=-v \gamma^{4} \Omega_{0}^{2}\left[\begin{array}{lll}
0 & 0 & 1 \\
0 & 0 & 0 \\
1 & 0 & 0
\end{array}\right] .
$$


It follows from Equation (29) that for $\tau \geq 0$, we have:

$$
\mathcal{F}_{ \pm}(\tau)=\left[1+i \mu \gamma^{2} \Omega_{0}^{2} \frac{e^{i \tilde{\omega}_{ \pm} \tau}-1}{\tilde{\omega}_{ \pm}}\right] \tilde{F}_{ \pm}(\tau)
$$

It is important to note that $\mathcal{F}_{ \pm}(\tau)$ can never become constant; moreover, for incident positive-helicity radiation with resonance frequency $\omega_{0}=\Omega_{0}$, we have $\tilde{\omega}_{+}=0$ and

$$
\mathcal{F}_{+}(\tau)=\left(1-\mu \gamma^{2} \Omega_{0}^{2} \tau\right) \tilde{F}_{+}
$$

where $\tilde{F}_{+}$is constant. In the measured field (56), the ratio of the positive-helicity wave amplitude to the negative-helicity wave amplitude is given by $\left(a_{+} / a_{-}\right) \rho$, where:

$$
\rho=\frac{\omega_{0}+\Omega_{0}}{\omega_{0}-\Omega_{0}} \frac{\omega_{0}-\Omega_{0}-i \mu \gamma \Omega_{0}^{2}}{\omega_{0}+\Omega_{0}-i \mu \gamma \Omega_{0}^{2}} .
$$

If $\mu=0$, then $\rho=1$, in accordance with the observation that the locality postulate does not affect the relative strength of the helicity amplitudes [48,49]. However, when $\mu \neq 0,|\rho|>1$, as expected; that is, for an observer that is rotating uniformly in the positive sense about the $z$ axis, the measured amplitude of the incident positive-helicity radiation is enhanced, while the measured amplitude of the negative-helicity radiation is diminished $[48,49,59]$. This result is independent of the sign and magnitude of $\mu \neq 0$.

\section{Rotating Observer in Constant Electromagnetic Field}

Consider a constant electromagnetic field in the background global inertial frame. Suppose that for $\tau>0$, the uniformly rotating observer measures the electromagnetic field. Thus in our nonlocal ansatz, Equation (29), we use the quadratic kernel (47) together with $\tilde{F}=\Lambda F$, where $F$ given in Equation (28) is constant and $\Lambda$ is given by Equation (50).

For the sake of simplicity, it is useful to define:

$$
C:=\cos \varphi, \quad S:=\sin \varphi, \quad \varphi=\Omega_{0} t=\gamma \Omega_{0} \tau
$$

and auxiliary dimensionless parameters $\mu^{\prime}$ and $\nu^{\prime}$ given by:

$$
\mu^{\prime}:=\gamma^{2} \Omega_{0} \mu, \quad v^{\prime}=2 \gamma \Omega_{0} \nu
$$

A detailed but straightforward calculation reveals that:

$$
\begin{gathered}
\mathcal{E}_{1}=\gamma\left(C E_{1}+S E_{2}\right)-\mu^{\prime}\left[S E_{1}+(1-C) E_{2}\right]+v \gamma\left(1+v^{\prime} \varphi\right) B_{3} \\
\mathcal{E}_{2}=-S E_{1}+C E_{2}+\gamma^{-1} \mu^{\prime}\left[(1-C) E_{1}-S E_{2}\right] \\
\mathcal{E}_{3}=\gamma E_{3}-v \gamma\left(C B_{1}+S B_{2}\right)+v \mu^{\prime}\left[S B_{1}+(1-C) B_{2}\right] \\
\mathcal{B}_{1}=\gamma\left(C B_{1}+S B_{2}\right)-\mu^{\prime}\left[S B_{1}+(1-C) B_{2}\right]-v \gamma E_{3} \\
\mathcal{B}_{2}=-S B_{1}+C B_{2}+\gamma^{-1} \mu^{\prime}\left[(1-C) B_{1}-S B_{2}\right] \\
\mathcal{B}_{3}=v \gamma\left(C E_{1}+S E_{2}\right)-v \mu^{\prime}\left[S E_{1}+(1-C) E_{2}\right]+\gamma\left(1+v^{\prime} \varphi\right) B_{3} .
\end{gathered}
$$

It is important to observe that while terms proportional to $\mu^{\prime}$ are harmonic, terms proportional to $v^{\prime}$ are secular. That is, as the observer rotates uniformly, the $\mu^{\prime}$ terms are simply periodic with proper period $2 \pi /\left(\gamma \Omega_{0}\right)$, but the $v^{\prime}$ terms are cumulative. If $B_{3} \neq 0$, then $\mathcal{E}_{1}$ and $\mathcal{B}_{3}$ can grow linearly with time. This is not physically acceptable and indicates that we should eventually drop the second term in the quadratic field kernel (i.e., $v=0$ ). 


\section{Linearly Accelerated Observer in Constant Field}

Consider next an observer moving with uniform acceleration along the $z$ axis for $t>0$. For $-\infty<$ $t<0$, the observer is at rest at the spatial origin of a Cartesian coordinate system $(x, y, z)$ in an inertial reference frame. The acceleration is turned on at $t=\tau_{0}=0$ and will be turned off at $\tau_{f}>0$. For $0 \leq \tau \leq \tau_{f}$, the tetrad frame of the observer in $(t, x, y, z)$ coordinates is given by:

$$
\begin{aligned}
& \lambda^{\mu}{ }_{\hat{0}}=(\mathbb{C}, 0,0, \mathbb{S}), \\
& \lambda^{\mu}{ }_{\hat{1}}=(0,1,0,0), \\
& \lambda^{\mu}{ }_{\hat{2}}=(0,0,1,0), \\
& \lambda^{\mu}{ }_{\hat{3}}=(\mathbb{S}, 0,0, \mathbb{C}),
\end{aligned}
$$

where

$$
\mathbb{C}=\cosh (g \tau), \quad \mathbb{S}=\sinh (g \tau) .
$$

Here, $g$ is the constant invariant translational acceleration of the observer, that is, the acceleration tensor in this case is given by $\mathbf{g}=g(0,0,1)$ and $\Omega=0$ with respect to the local adapted spatial frame $\lambda^{\mu} \hat{i}^{\prime} i=1,2,3$, that indicate the Cartesian axes $x, y$, and $z$ directions, respectively. The observer's Lorentz factor and speed are given by $\mathbb{C}$ and $\mathbb{S} / \mathbb{C}$, respectively.

The background field $F$ given by Equation (28) is again constant. The field as measured by the instantaneously tangent inertial observer is $\tilde{F}=\Lambda F$, where $\Lambda$ has the same form as in Equation (50), but with $\Lambda_{1}$ and $\Lambda_{2}$ is given by:

$$
\Lambda_{1}=\operatorname{diag}(\mathbb{C}, \mathbb{C}, 1), \quad \Lambda_{2}=-\mathbb{S} I_{3}
$$

The special kernel $\kappa$ given by Equation (38) reduces in this case to:

$$
\kappa_{1}=0, \quad \kappa_{2}=g I_{3}, \quad I_{3}=\left[\begin{array}{ccc}
0 & -1 & 0 \\
1 & 0 & 0 \\
0 & 0 & 0
\end{array}\right] .
$$

In the formula for $\mathbb{K}$, we need $-\kappa_{2}^{2}=g^{2} \operatorname{diag}(1,1,0)$; hence,

$$
2 \mathbb{K}=g^{2} \operatorname{diag}(\mu, \mu,-2 v, \mu, \mu, 0) .
$$

As before, a detailed but straightforward calculation based on Equation (29) reveals that:

$$
\begin{gathered}
\mathcal{E}_{1}=\mathbb{C} E_{1}-\mathbb{S} B_{2}+\mu g\left[\mathbb{S} E_{1}-(\mathbb{C}-1) B_{2}\right], \\
\mathcal{E}_{2}=\mathbb{C} E_{2}+\mathbb{S} B_{1}+\mu g\left[\mathbb{S} E_{2}+(\mathbb{C}-1) B_{1}\right], \\
\mathcal{E}_{3}=\left(1-2 v g^{2} \tau\right) E_{3}, \\
\mathcal{B}_{1}=\mathbb{C} B_{1}+\mathbb{S} E_{2}+\mu g\left[\mathbb{S} B_{1}+(\mathbb{C}-1) E_{2}\right], \\
\mathcal{B}_{2}=\mathbb{C} B_{2}-\mathbb{S} E_{1}+\mu g\left[\mathbb{S} B_{2}-(\mathbb{C}-1) E_{1}\right], \\
\mathcal{B}_{3}=B_{3} .
\end{gathered}
$$

The only contribution from the second term in the quadratic kernel (47) occurs in $\mathcal{E}_{3}=(1-$ $\left.2 v g^{2} \tau\right) E_{3}$, which is again cumulative. If $E_{3} \neq 0$, then the measured magnitude of the electric field along the direction of motion varies linearly with proper time. As before, it seems appropriate to set $v=0$. 


\section{Discussion}

It is noteworthy that parity violating terms had to be introduced for the sake of consistency in the theories of acceleration-induced nonlocal electrodynamics as well as nonlocal gravity [6]. On the other hand, it is important to understand theoretically why their presence was deemed necessary; that is, one has to find out whether these were genuine or somehow spurious and a consequence of some assumption that had no firm physical foundation. It is simpler to look at the problem in nonlocal electrodynamics, where the kernel was originally assumed to be linear in the acceleration tensor. Removing this restriction has been the main objective of the present work. We find that the kernel could in principle contain linear, quadratic, and possibly higher-order parity conserving terms in the acceleration tensor. Concentrating on quadratic kernels, we have worked out some of the physical consequences of an acceleration-induced nonlocal field theory of electrodynamics that involves a kernel given by:

$$
K_{\hat{\gamma} \hat{\alpha} \hat{\delta}}^{\hat{\alpha} \hat{\beta}}{ }_{\hat{\rho} \hat{\sigma}}^{\hat{\alpha} \hat{\beta}} \kappa_{\hat{\rho} \hat{\sigma} \hat{\delta}}
$$

where $\mu$ is a constant parameter with dimensions of length that must be determined from comparison of the theory with observational data.

What are the implications of the present investigation for the problem of parity violation in nonlocal gravity? Let us note that general relativity (GR) has an equivalent tetrad formulation as a teleparallel theory of gravity $\left(G_{\|}\right)$. Nonlocal gravity is indeed the nonlocal version of GR ${ }_{\|}$, the teleparallel equivalent of GR. Specifically, consider a tetrad field $e_{\hat{\alpha}}^{\mu}(x)$ adapted to a set of preferred observers in spacetime. The tetrad system is orthonormal, i.e.,

$$
g^{\mu \nu}=\eta^{\hat{\alpha} \hat{\beta}} e_{\hat{\alpha}}^{\mu} e_{\hat{\beta}}^{v}
$$

which defines the spacetime metric tensor $g_{\mu \nu}(x)$. The gravitational field in this framework is given by the torsion tensor:

$$
C_{\mu \nu}^{\hat{\alpha}}=\partial_{\mu} e_{\nu}^{\hat{\alpha}}-\partial_{\nu} e_{\mu}^{\hat{\alpha}}
$$

which, for each $\alpha=0,1,2,3$, bears a strong resemblance to the electromagnetic field tensor. Using the torsion tensor, one can define three independent algebraic invariants given by:

$$
\mathcal{I}_{1}=C_{\alpha \beta \gamma} C^{\alpha \beta \gamma}, \quad \mathcal{I}_{2}=C_{\alpha \beta \gamma} C^{\gamma \beta \alpha}, \quad \mathcal{I}_{3}=C_{\alpha} C^{\alpha} \text {, }
$$

where $C_{\alpha}$ is the torsion vector: $C_{\alpha}=C_{\beta \alpha}{ }^{\beta}=-C_{\alpha}{ }_{\beta}$. With a Lagrangian density:

$$
\mathcal{L}_{g}=-\frac{c^{3}}{32 \pi G} \sqrt{-g}\left(\frac{1}{2} \mathcal{I}_{1}+\mathcal{I}_{2}-2 \mathcal{I}_{3}\right),
$$

we recover the field equations of $\mathrm{GR}_{\|}$, which exhibit formal similarities with the Maxwell field equations in a material medium. In the nonlocal electrodynamics of media, nonlocality enters the theory via constitutive relations. The same idea can be implemented in nonlocal gravity, that is, the local constitutive relation of $\mathrm{GR}_{||}$can be rendered nonlocal via the introduction of a scalar kernel. The resulting theory has only one known trivial exact solution, namely, the Minkowski spacetime in the absence of gravitation. It has not been possible thus far to find any nontrivial exact solution of the theory [80]; instead, linearized $\mathrm{GR}_{||}$has been studied in detail [6]. As elucidated in [6], the equations of linearized nonlocal gravity exhibit a certain inconsistency that can be eliminated by adding to the constitutive relation a parity violating term proportional to:

$$
\check{C}_{\mu} g_{\nu \rho}-\check{C}_{\nu} g_{\mu \rho}
$$


where $\check{C}_{\mu}$ is the torsion pseudovector given by:

$$
\check{C}_{\mu}=\frac{1}{3 !} C^{\alpha \beta \gamma} \epsilon_{\alpha \beta \gamma \mu} .
$$

General discussions of parity violation in tetrad theories of gravitation are contained in $[81,82]$. In connection with nonlocal $\mathrm{GR}_{\|}$, various theoretical aspects of parity violating term (86) have been explored in [82]. Can one avoid parity violation in nonlocal gravity as in the nonlocal electrodynamics of accelerated systems? The resolution of this issue requires further investigation.

Funding: This research received no external funding.

Acknowledgments: Discussions initiated by Friedrich W. Hehl about parity violation in nonlocal gravity motivated the present investigation. I am also grateful to him for his insightful and critical comments.

Conflicts of Interest: The author declares no conflict of interest.

\section{Appendix A. $P$ and $T$ Invariance in Standard Electrodynamics of Accelerated Systems and Gravitational Fields}

It can be shown that the electromagnetic field equations in an accelerated system or a gravitational field can be reformulated as Maxwell's equations in flat Minkowski spacetime in Cartesian coordinates but in the presence of a certain hypothetical "medium". To this end, imagine Maxwell's equations:

$$
F_{[\mu v, \rho]}=0, \quad\left(\sqrt{-g} F^{\mu v}\right)_{, v}=4 \pi j^{\mu}
$$

on a background spacetime metric $-d s^{2}=g_{\mu \nu} d x^{\mu} d x^{\nu}$ expressed in standard Cartesian coordinates $x^{\mu}=(t, x, y, z)$. We use the natural decompositions:

$$
F_{\mu v} \mapsto(\mathbf{E}, \mathbf{B}), \quad \sqrt{-g} F^{\mu v} \mapsto(-\mathbf{D}, \mathbf{H}),
$$

where $\sqrt{-g} F^{0 i}=D_{i}$ and $\sqrt{-g} F^{i j}=\epsilon^{i j k} H_{k}$ in our convention. In this way, we recover the original form of Maxwell's equations in Minkowski spacetime but in a background gyrotropic medium with constitutive relations [83-86]:

$$
D_{i}=\epsilon_{i j} E_{j}-(\mathbf{G} \times \mathbf{H})_{i}, \quad B_{i}=\mu_{i j} H_{j}+(\mathbf{G} \times \mathbf{E})_{i},
$$

where the conformally invariant properties of the optical medium are given by equal dielectric and permeability tensors as well as a gyration vector which has the interpretation of the gravitational vector potential,

$$
\epsilon_{i j}=\mu_{i j}=-\sqrt{-g} \frac{g^{i j}}{g_{00}}, \quad G_{i}=-\frac{g_{0 i}}{g_{00}} .
$$

We briefly discuss the derivation of the constitutive relations (A3) at the end of this appendix. There is no double refraction when the electric permittivity and magnetic permeability tensors coincide, which is a special property of the medium that simulates acceleration and gravitation in this case [86].

Under the parity transformation $P:(x, y, z) \mapsto(-x,-y,-z)$ or time reversal $T: t \mapsto-t$, the background spacetime interval remains invariant, while the components of the metric tensor transform as $\left(g_{00}, g_{0 i}, g_{i j}\right) \mapsto\left(g_{00},-g_{0 i}, g_{i j}\right)$. Similarly, we find for the inverse metric $\left(g^{00}, g^{0 i}, g^{i j}\right) \mapsto$ $\left(g^{00},-g^{0 i}, g^{i j}\right)$. Furthermore, we can write:

$$
-d s^{2}=g_{00}\left(d t-G_{k} d x^{k}\right)^{2}+\gamma_{i j} d x^{i} d x^{j}
$$

where $\left(\gamma_{i j}\right)$, defined by:

$$
\gamma_{i j}:=g_{i j}-\frac{g_{0 i} g_{0 j}}{g_{00}}
$$


is the inverse of $\left(g^{i j}\right)$; that is, $g^{i k} \gamma_{k j}=\delta_{j}^{i}$. For the determinant of the metric tensor $g:=\operatorname{det}\left(g_{\mu v}\right)$, we have the general relation $g=g_{00} \operatorname{det}\left(\gamma_{i j}\right)$. Moreover,

$$
g_{00}=g \operatorname{det}\left(g^{i j}\right), \quad g^{00}=\frac{\operatorname{det}\left(g_{i j}\right)}{g},
$$

which imply that $g$ does not change under parity and time reversal. These results are all consistent with the fact that under parity and time reversal, we have $\left(\mu_{i j}, \mathbf{G}_{i}\right) \mapsto\left(\mu_{i j},-\mathbf{G}_{i}\right)$. Maxwell's equations in an inertial frame in Minkowski spacetime are such that under parity $\mathbf{E}$ and $\mathbf{D}$ transform as vectors, while $\mathbf{B}$ and $\mathbf{H}$ transform as pseudovectors. Moreover, under time reversal we have $(\mathbf{E}, \mathbf{B}) \mapsto(\mathbf{E},-\mathbf{B})$ and $(\mathbf{D}, \mathbf{H}) \mapsto(\mathbf{D},-\mathbf{H})$. The constitutive relations (A3) remain unchanged separately under these parity and time reversal transformations. Therefore, parity is conserved and time reversal invariance is maintained in the standard electrodynamics of accelerated systems and gravitational fields.

It is interesting to write the general Maxwell equations in Dirac form. For this purpose, we introduce the Riemann-Silberstein vectors:

$$
\mathbf{F}^{ \pm}=\mathbf{E} \pm i \mathbf{H}, \quad \mathbf{S}^{ \pm}=\mathbf{D} \pm i \mathbf{B}
$$

in terms of complex fields, so that Maxwell's equations can now be written as [87-90]:

$$
\frac{1}{i} \nabla \times \mathbf{F}^{ \pm}= \pm \frac{\partial \mathbf{S}^{ \pm}}{\partial t} \pm 4 \pi \mathbf{J}, \quad \nabla \cdot \mathbf{S}^{ \pm}=4 \pi \rho,
$$

where $j^{\mu}=(\rho, \mathbf{J})$ and

$$
S_{p}^{ \pm}=\mu_{p q} F_{q}^{ \pm} \pm i\left(\mathbf{G} \times \mathbf{F}^{ \pm}\right)_{p} .
$$

This formalism has been explicitly employed in [8] to study electromagnetic wave propagation in a rotating frame of reference.

The parity operation can also be applied to the Dirac form of Maxwell's equations given by Equations (A9) and (A10). For this purpose, we must think of $\pm i \mathbf{B}$ and $\pm i \mathbf{H}$ as vectors and $-i \mathbf{E}$ as a pseudovector even though $\mathbf{B}$ and $\mathbf{H}$ are axial vectors and $\mathbf{E}$ is a vector. Similarly, under time reversal, we must assume that $\pm i \mathbf{B}$ and $\pm i \mathbf{H}$ remain invariant, while $-i \mathbf{E}$ changes sign. In this way, the fields become consistent with sources under parity and time reversal operations; that is, we have $\rho \mapsto \rho$ and $J \mapsto-J$ separately under $P$ and $T$.

An interesting consequence of $\epsilon_{i j}=\mu_{i j}$ should be noted here: Under duality rotations with constant $\alpha$, namely,

$$
\mathbf{E}^{\prime}=\mathbf{E} \cos \alpha+\mathbf{H} \sin \alpha, \quad \mathbf{D}^{\prime}=\mathbf{D} \cos \alpha+\mathbf{B} \sin \alpha
$$

and

$$
\mathbf{H}^{\prime}=-\mathbf{E} \sin \alpha+\mathbf{H} \cos \alpha, \quad \mathbf{B}^{\prime}=-\mathbf{D} \sin \alpha+\mathbf{B} \cos \alpha,
$$

constitutive relations (A3) and Maxwell's equations in the absence of external sources remain invariant; in this connection, let us observe that $\mathbf{F}^{\prime \pm}=\mathbf{F}^{ \pm} e^{\mp i \alpha}$ and $\mathbf{S}^{\prime \pm}=\mathbf{S}^{ \pm} e^{\mp i \alpha}$ under the duality rotations.

\section{Derivation of the Constitutive Relations}

For the sake of completeness, we briefly indicate here the derivation of constitutive relations (A3), which follow from the electric and magnetic parts of:

$$
\sqrt{-g} F_{\mu \nu}=g_{\mu \alpha} g_{\nu \beta} \sqrt{-g} F^{\alpha \beta}, \quad \sqrt{-g} F^{\mu v}=\sqrt{-g} g^{\mu \alpha} g^{\nu \beta} F_{\alpha \beta},
$$

respectively. The electric part of the first relation in Equation (A13) implies:

$$
-\sqrt{-g} E_{j}=g_{00} \gamma_{j k} D_{k}-g_{j p} g_{0 q} \epsilon^{p q n} H_{n} .
$$


We multiply both sides of this equation by $g^{i j} / g_{00}$ and use $g^{i j} \gamma_{j k}=\delta_{k}^{i}$ as well as $g^{i j} g_{j p}=\delta_{p}^{i}-g^{0 i} g_{0 p}$ to obtain the first constitutive relation.

Next, the magnetic part of the second relation in Equation (A13) can be written as:

$$
H_{j}=\sqrt{-g} \epsilon_{j m n}\left[g^{m p} g^{0 n} E_{p}+\frac{1}{2} \epsilon_{a b c} g^{m a} g^{n b} B_{c}\right] .
$$

Starting with $\left(g^{\mu \nu}\right)$, one can determine the inverse matrix element $g_{0 k}$ via:

$$
g \epsilon_{a b c} g^{a i} g^{b j} g^{0 c}=-\epsilon^{i j k} g_{0 k} .
$$

Furthermore, from the definition of the determinant and Equation (A7) we find:

$$
\epsilon_{a b c} g^{a i} g^{b j} g^{c k}=\epsilon^{i j k} \operatorname{det}\left(g^{p q}\right)=\epsilon^{i j k} \frac{g_{00}}{g} .
$$

Multiplying both sides of Equation (A15) by $-\sqrt{-g} g^{i j} / g_{00}$ and using Equations (A16) and (A17), we get the second constitutive relation.

\section{References}

1. Einstein, A. The Meaning of Relativity; Princeton University Press: Princeton, NJ, USA, 1955.

2. Mashhoon, B. Limitations of spacetime measurements. Phys. Lett. A 1990, 143, 176-182. [CrossRef]

3. Mashhoon, B. The hypothesis of locality in relativistic physics. Phys. Lett. A 1990, 145, 147-153. [CrossRef]

4. Maluf, J.W.; Ulhoa, S.C. Electrodynamics in accelerated frames revisited. Ann. Phys. (Berlin) 2010, 522, 766-775. [CrossRef]

5. Maluf, J.W.; Faria, F.F. The electromagnetic field in accelerated frames. arXiv 2011, arXiv:1110.5367 [gr-qc].

6. Mashhoon, B. Nonlocal Gravity; Oxford University Press: Oxford, UK, 2017.

7. Mashhoon, B. General covariance and quantum theory. Found. Phys. (Wheeler Festschrift) 1986, 16, 619-635 (Reprinted in Between Quantum and Cosmos; Zurek, W.H., van der Merwe, A., Miller, W.A., Eds.; Princeton University Press: Princeton, NJ, USA, 1988). [CrossRef]

8. Hauck, J.C.; Mashhoon, B. Electromagnetic waves in a rotating reference frame. Ann. Phys. (Berlin) 2003, 12, 275-288. [CrossRef]

9. Mashhoon, B. Modification of the Doppler effect due to the helicity-rotation coupling. Phys. Lett. A 2002, 306, 66-72. [CrossRef]

10. Ashby, N. Relativity in the Global Positioning System. Living Rev. Relativ. 2003, 6, 1. [CrossRef]

11. Allen, P.J. A radiation torque experiment. Am. J. Phys. 1966, 34, 1185-1192. [CrossRef]

12. Mashhoon, B.; Neutze, R.; Hannam, M.; Stedman, G.E. Observable frequency shifts via spin-rotation coupling. Phys. Lett. A 1998, 249, 161-166. [CrossRef]

13. Mashhoon, B.; Kaiser, H. Inertia of intrinsic spin. Physica B 2006, 385-386, 1381-1383. [CrossRef]

14. Mashhoon, B. Electrodynamics in a rotating frame of reference. Phys. Lett. A 1989, 139, 103-108. [CrossRef]

15. Anderson, J.D.; Mashhoon, B. Pioneer anomaly and the helicity-rotation coupling. Phys. Lett. A 2003, 315, 199-202. [CrossRef]

16. Mashhoon, B. Optics of rotating systems. Phys. Rev. A 2009, 79, 062111. [CrossRef]

17. Bliokh, K.Y.; Rodríguez-Fortuño, F.J.; Nori, F.; Zayats, A.V. Spin-orbit interactions of light. Nat. Photon 2015, 9, 796-808. [CrossRef]

18. Mashhoon, B. Neutron interferometry in a rotating frame of reference. Phys. Rev. Lett. 1988, 61, $2639-2642$. [CrossRef]

19. Hehl, F.W.; Ni, W.-T. Inertial effects of a Dirac particle. Phys. Rev. D 1990, 42, 2045-2048. [CrossRef]

20. Mashhoon, B. Reply to comment on spin-rotation-gravity coupling. Phys. Rev. Lett. 1992, 68, 3812. [CrossRef]

21. Soares, I.D.; Tiomno, J. The physics of the Sagnac-Mashhoon effects. Phys. Rev. D 1996, 54, $2808-2813$. [CrossRef]

22. Ryder, L. Relativistic treatment of inertial spin effects. J. Phys. A 1998, 31, 2465-2469. [CrossRef] 
23. Singh, D.; Papini, G. Spin-1/2 particles in non-inertial reference frames: low-and high-energy approximations. Nuovo Cimento B 2000, 115, 223-238.

24. Papini, G. Parity and time reversal in the spin-rotation interaction. Phys. Rev. D 2002, 65, 077901. [CrossRef]

25. Shen, J.-Q.; He, S.-L. Geometric phases of electrons due to spin-rotation coupling in rotating $\mathrm{C}_{60}$ molecules. Phys. Rev. B 2003, 68, 195421. [CrossRef]

26. Lambiase, G.; Papini, G. Discrete symmetries in the spin-rotation interaction. Phys. Rev. D 2004, 70, 097901. [CrossRef]

27. Randono, A. Do spinors give rise to a frame-dragging effect? Phys. Rev. D 2010, 81, 024027. [CrossRef]

28. Pan, Y.Y.; Ren, J. Spin-rotation coupling in the teleparallelism description in high speed rotation system. Int. J. Theor. Phys. 2011, 50, 724-728. [CrossRef]

29. Arminjon, M. On the non-uniqueness problem of the covariant Dirac theory and the spin-rotation coupling. Int. J. Theor. Phys. 2013, 52, 4032-4044. [CrossRef]

30. Werner, S. Does a neutron know that the earth is rotating? Gen. Relativ. Gravit. 2008, 40, 921-934. [CrossRef]

31. Rauch, H.; Werner, S.A. Neutron Interferometry, 2nd ed.; Oxford University Press: Oxford, UK, 2015.

32. Demirel, B.; Sponar, S.; Hasegawa, Y. Measurement of the spin-rotation coupling in neutron polarimetry. New J. Phys. 2015, 17, 023065. [CrossRef]

33. Danner, A.; Demirel, B.; Sponar, S.; Hasegawa, Y. Development and performance of a miniaturised spin rotator suitable for neutron interferometer experiments. J. Phys. Commun. 2019, 3, 035001. [CrossRef]

34. Danner, A.; Demirel, B.; Kersten, W.; Wagner, R.; Lemmel, H.; Sponar, S.; Hasegawa, Y. Spin-rotation coupling observed in neutron interferometry. Npj Quantum Inf. 2020, 6, 23. [CrossRef]

35. Matsuo, M.; Ieda, J.; Saitoh, E.; Maekawa, S. Effects of mechanical rotation on spin currents. Phys. Rev. Lett. 2011, 106, 076601. [CrossRef] [PubMed]

36. Matsuo, M.; Ieda, J.; Saitoh, E.; Maekawa, S. Spin-dependent inertial force and spin current in accelerating systems. Phys. Rev. B 2011, 84, 104410. [CrossRef]

37. Matsuo, M.; Ieda, J.; Harii, K.; Saitoh, E.; Maekawa, S. Mechanical generation of spin current by spin-rotation coupling. Phys. Rev. B 2013, 87, 180402. [CrossRef]

38. Matsuo, M.; Ieda, J.; Maekawa, S. Renormalization of spin-rotation coupling. Phys. Rev. B 2013, 87, 115301. [CrossRef]

39. Chowdhury, D.; Basu, B. Effect of spin rotation coupling on spin transport. Ann. Phys. 2013, 339, 358-370. [CrossRef]

40. Ieda, J.; Matsuo, M.; Maekawa, S. Theory of mechanical spin current generation via spin-rotation coupling. Solid State Commun. 2014, 198, 52-56. [CrossRef]

41. Papini, G. Spin currents in non-inertial frames. Phys. Lett. A 2013, 377, 960-963. [CrossRef]

42. Kobayashi, D.; Yoshikawa, T.; Matsuo, M.; Iguchi, R.; Maekawa, S.; Saitoh, E.; Nozaki, Y. Spin current generation using a surface acoustic wave generated via spin-rotation coupling. Phys. Rev. Lett. 2017, 119, 077202. [CrossRef]

43. Kurimune, Y.; Matsuo, M.; Nozaki, Y. Observation of gyromagnetic spin wave resonance in NiFe films. Phys. Rev. Lett. 2020, 124, 217205. [CrossRef]

44. Nakata, K.; Takayoshi, S. Optomagnonic Barnett effect. Phys. Rev. B 2020, 102, 094417. [CrossRef]

45. Kurimune, Y.; Matsuo, M.; Maekawa, S.; Nozaki, Y. Highly nonlinear frequency-dependent spin-wave resonance excited via spin-vorticity coupling. Phys. Rev. B 2020, 102, 174413. [CrossRef]

46. Schilpp, P.A. Albert Einstein: Philosopher-Scientist; Library of Living Philosophers: Evanston, IL, USA, 1949.

47. Mashhoon, B. Nonlocal theory of accelerated observers. Phys. Rev. A 1993, 47, 4498-4501. [CrossRef] [PubMed]

48. Mashhoon, B. Nonlocal electrodynamics of rotating systems. Phys. Rev. A 2005, 72, 052105. [CrossRef]

49. Mashhoon, B. Nonlocal special relativity. Ann. Phys. (Berlin) 2008, 17, 705-727. [CrossRef]

50. Bremm, G.N.; Falciano, F.T. Nonlocal effects in black body radiation. Ann. Phys. (Berlin) 2015, 527, $265-277$. [CrossRef]

51. Bohr, N.; Rosenfeld, L. Zur Frage der Messbarkeit der elektromagnetischen Feldgrössen. K. Dan. Vidensk. Selsk. Mat. Fys. Medd. 1933, 12, 8. (English translation: On the question of measurability of electromagnetic field quantities. In Quantum Theory and Measurement; Wheeler, J.A., Zurek, W.H., Eds.; Princeton University Press: Princeton, NJ, USA, 1983). 
52. Bohr, N.; Rosenfeld, L. Field and charge measurements in quantum electrodynamics. Phys. Rev. 1950, 78, 794-798. [CrossRef]

53. Volterra, V. Theory of Functionals and of Integral and Integro-Differential Equations; Dover: New York, NY, USA, 1959.

54. Tricomi, F.G. Integral Equations; Interscience: New York, NY, USA, 1957.

55. Mashhoon, B. Nonlocal Dirac equation for accelerated observers. Phys. Rev. A 2007, 75, 042112. [CrossRef]

56. Kennard, E.H. On unipolar induction: Another experiment and its significance as evidence for the existence of the æther. Phil. Mag. 1917, 33, 179-190. [CrossRef]

57. Pegram, G.B. Unipolar induction and electron theory. Phys. Rev. 1917, 10, 591-600. [CrossRef]

58. Swann, W.F.G. Unipolar induction. Phys. Rev. 1920, 15, 365-398. [CrossRef]

59. Mashhoon, B. Nonlocal electrodynamics of accelerated systems. Phys. Lett. A 2007, 366, 545-549. [CrossRef]

60. Muench, U.; Hehl, F.W.; Mashhoon, B. Acceleration-induced nonlocal electrodynamics in Minkowski spacetime. Phys. Lett. A 2000, 271, 8-15. [CrossRef]

61. Hehl, F.W.; Obukhov, Y.N. Foundations of Classical Electrodynamics: Charge, Flux, and Metric; Birkhäuser: Boston, MA, USA, 2003.

62. Hehl, F.W.; Obukhov, Y.N.; Rivera, J.P.; Schmid, H. Relativistic analysis of magnetoelectric crystals: Extracting a new 4-dimensional $P$ odd and $T$ odd pseudoscalar from $\mathrm{Cr}_{2} \mathrm{O}_{3}$ data. Phys. Lett. A 2008, 372, 1141-1146. [CrossRef]

63. Hehl, F.W.; Obukhov, Y.N.; Rivera, J.P.; Schmid, H. Relativistic nature of a magnetoelectric modulus of $\mathrm{Cr}_{2} \mathrm{O}_{3}$ crystals: A four-dimensional pseudoscalar and its measurement. Phys. Rev. A 2008, 77, 022106. [CrossRef]

64. Hehl, F.W.; Obukhov, Y.N.; Rivera, J.P.; Schmid, H. Magnetoelectric $\mathrm{Cr}_{2} \mathrm{O}_{3}$ and relativity theory. Eur. Phys. J. B 2009, 71, 321-329. [CrossRef]

65. Essin, A.M.; Moore, J.E.; Vanderbilt, D. Magnetoelectric polarizability and axion electrodynamics in crystalline insulators. Phys. Rev. Lett. 2009, 102, 146805. [CrossRef]

66. Stueckelberg, E.C.G. Violation of parity conservation and general relativity. Phys. Rev. 1957, 106, 388-389. [CrossRef]

67. Kobzarev, I.Y.; Okun, L.B. Gravitational interaction of fermions. Sov. Phys.-JETP 1963, 16, 1343-1346.

68. Leitner, J.; Okubo, S. Parity, charge conjugation, and time reversal in the gravitational interaction. Phys. Rev. 1964, 136, B1542-B1546. [CrossRef]

69. Wineland, D.J.; Ramsey, N.F. Atomic deuterium maser. Phys. Rev. A 1972, 5, 821-837. [CrossRef]

70. Mashhoon, B. On the coupling of intrinsic spin with the rotation of the earth. Phys. Lett. A 1995, 198, 9-13. [CrossRef]

71. Mashhoon, B. Gravitational couplings of intrinsic spin. Class. Quantum Gravity 2000, 17, $2399-2409$. [CrossRef]

72. Freidel, L.; Minic, D.; Takeuchi, T. Quantum gravity, torsion, parity violation and all that. Phys. Rev. D 2005, 72, 104002. [CrossRef]

73. Ivanov, A.N.; Snow, W.M. Parity-even and time-reversal-odd neutron optical potential in spinning matter induced by gravitational torsion. Phys. Lett. B 2017, 764, 186-189. [CrossRef]

74. Conroy, A.; Koivisto, T. Parity-violating gravity and GW170817 in non-Riemannian cosmology. J. Cosmol. Astropart. Phys. 2019, 12, 016. [CrossRef]

75. Zhao, W.; Zhu, T.; Qiao, J.; Wang, A. Waveform of gravitational waves in the general parity-violating gravities. Phys. Rev. D 2020, 101, 024002. [CrossRef]

76. Iosifidis, D.; Ravera, L. Parity violating metric-affine gravity theories. arXiv 2020, arXiv:2009.03328 [gr-qc].

77. Obukhov, Y.N. Parity violation in Poincaré gauge gravity. arXiv 2020, arXiv:2010.16276 [gr-qc].

78. Hehl, F.W.; Mashhoon, B. Nonlocal gravity simulates dark matter. Phys. Lett. B 2009, 673, 279-282. [CrossRef]

79. Hehl, F.W.; Mashhoon, B. Formal framework for a nonlocal generalization of Einstein's theory of gravitation. Phys. Rev. D 2009, 79, 064028. [CrossRef]

80. Bini, D.; Mashhoon, B. Nonlocal gravity: Conformally flat spacetimes. Int. J. Geom. Meth. Mod. Phys. 2016, 13, 1650081. [CrossRef]

81. Baekler, P.; Hehl, F.W. Beyond Einstein-Cartan gravity: Quadratic torsion and curvature invariants with even and odd parity including all boundary terms. Class. Quantum Gravity 2011, 28, 215017. [CrossRef]

82. Itin, Y.; Obukhov, Y.N.; Boos, J.; Hehl, F.W. Premetric teleparallel theory of gravity and its local and linear constitutive law. Eur. Phys. J. C 2018, 78, 907. [CrossRef] 
83. Skrotskii, G.V. The influence of gravitation on the propagation of light. Sov. Phys. Doklady 1957, 2, $226-229$.

84. Plebanski, J. Electromagnetic waves in gravitational fields. Phys. Rev. 1960, 118, 1396-1408. [CrossRef]

85. de Felice, F. On the gravitational field acting as an optical medium. Gen. Relativ. Gravit. 1971, 2, 347-357. [CrossRef]

86. Volkov, A.M.; Izmest'ev, A.A.; Skrotskii, G.V. The propagation of electromagnetic waves in a Riemannian space. Sov. Phys. JETP 1971, 32, 686.

87. Mashhoon, B. Scattering of electromagnetic radiation from a black hole. Phys. Rev. D 1973, 7, $2807-2814$. [CrossRef]

88. Mashhoon, B. Electromagnetic scattering from a black hole and the glory effect. Phys. Rev. D 1974, 10, 1059-1063. [CrossRef]

89. Mashhoon, B. Can Einstein's theory of gravitation be tested beyond the geometrical optics limit? Nature 1974, 250, 316. [CrossRef]

90. Mashhoon, B. Influence of gravitation on the propagation of electromagnetic radiation. Phys. Rev. D 1975, 11, 2679-2684. [CrossRef]

Publisher's Note: MDPI stays neutral with regard to jurisdictional claims in published maps and institutional affiliations.

(C) 2020 by the author. Licensee MDPI, Basel, Switzerland. This article is an open access article distributed under the terms and conditions of the Creative Commons Attribution (CC BY) license (http:/ / creativecommons.org/licenses/by/4.0/). 